\section{Novamente é Final de Ano: Hora de Agradecer e Recordar}

$\mathrm{N}$ EM BEM TENHO organizado meus encargos assumidos no início do ano e já me vejo obrigado a elaborar outro editorial de encerramento de ano. Este, como sempre, traz um sentimento dúbio, de tristeza e de alegria. De tristeza, porque mais um ano passou voando e, obviamente, muitas coisas ficaram por fazer. De alegria, porque um novo ano se apresenta, com suas perspectivas e possibilidades. Além disso, ao rememorar este ano de 2004 (junto com o de 2003, que parece ter passado despercebido, em termos dos agradecimentos devidos), vêm-me à cabeça muitas coisas boas e poucas, se alguma, ruins. Portanto, o balanço preliminar é de que a alegria deve predominar. Vamos ver.

Depois de um bom preparo de documentações durante 2003, começamos 2004 com a excelente notícia de que os ABE\&M estavam aceitos para indexação no sistema MEDLARS (Index Medicus, Medline, PubMed), o que, convenhamos, não foi um mau início de ano. Neste quesito, quero agradecer a várias pessoas que participaram ativamente do processo e outras que colaboraram escrevendo cartas de apoio e recomendação, que certamente foram cruciais na conquista deste ansiosamente aguardado desejo: Antonio C. Bianco, Valéria Guimarães, Leslie De Groot, John Bilezikian, Shlomo Melmed, Ilan Irony, Georges Chrousos, Geraldo Medeiros-Neto, Rui Maciel, Antonio Roberto Chacra, Amélio de Godoy Matos, entre outros.

Gosto de enfatizar nos agradecimentos de final de ano, a fundamental participação dos nossos autores-colaboradores. O número de submissões em 2003 atingiu 113 artigos, cerca de $50 \%$ a mais que a média dos anos anteriores. No entanto, 2004 - que ainda nem acabou -, superou todas as expectativas: mais de 200 artigos já foram cadastrados até $13 / 12 / 2004$. Esta oferta de manuscritos permitiu-nos a oportunidade de publicar 101 artigos em 2003 (excluindo cartas ao editor), dos quais 14 editoriais. Em 2004, foram publicados 119 artigos, sendo 16 editoriais. A média dos três anos anteriores ficou entre 60 e 70 artigos anuais. Portanto, nossos sinceros agradecimentos aos colegas que têm prestigiado a revista $\mathrm{e}$ que vislumbraram nela a melhor opção para suas publicações.

Como não poderia deixar de ser, agradeço em seguida àqueles que tornaram possível a publicação de tanto material de boa qualidade: nossos qualificados revisores do Conselho Editorial. Todos eles, recomendados a zelar pela preservação da qualidade científica da revista, têm dado muito de seu tempo lendo, corrigindo e sugerindo modificações nos trabalhos de outros colegas. Gostaria, assim, de agradecer individualmente àqueles que nos auxiliaram durante o biênio 2003-2004 (em ordem alfabética de primeiro nome): Adriana Costa e Forti, Alexander Jorge, Alfredo Halpern, Amanda Athayde, Amélio G. Matos, Ana Claudia Latronico, Ana Claudia Ramalho, Ana Luiza Maia, Ana Lydia Sawaia, Ana Maria Lengyel, André F. Reis, Andréa Brito-Fioretti, Ângela Reichelt, Antonio C. Bianco, Antonio C. Lerario, Antonio H. Lancha Jr., Antonio R. Chacra, Antonio S. Tebexreni, Ayrton C. Moreira, Balduino Tschiedel, Bruno Geloneze Neto, Carlos A. editorial

Claudio E. Kater
Professor Adjunto de Medicina,

Disciplina de Endocrinologia,

Departamento de Medicina,

Universidade Federal de São Paulo

(UNIFESP). Editor-chefe,

ABE\& $M$ 
Longui, Cármen Pazos-Moura, Catarina S. Porto, César Hayashida, César L. Boguszewski, Claudio Albino, Claudio E. Kater, Dalva M. Rocha, Daniel Giannella Neto, Daniel Lerario, Décio Mion, Denise Coitinho, Denise P. Carvalho, Dolores P. Pardini, Domingos Malerbi, Doris Rosenthal, Durval Damiani, Eder C. Quintão, Edmund C. Barakat, Edna Nakandakare, Eduardo Tomimori, Fabiano Sandrini, Flávio Zelmanovitz, Francisco Bandeira, Francisco Freire, Gabriela L. Saraiva, Geraldo Medeiros-Neto, Gil Guerra Jr., Gilberto Alonso, Gisah M. Amaral, Hans Graf, Helena Schmid, Hélio Bisi, Henrique L. Suplicy, Hermelinda C. Pedrosa, Hilton Kuperman, Hugo Lisboa, Ieda Verreschi, Ivaldir S. Dalbosco, Ivani Novato Silva, Ivo J.P. Arnhold, Jader B. Ferreira, Jairo T. Hidal, João Carlos Dias, João Hamilton Romaldini, João Roberto de Sá, João Roberto M. Martins, Jorge L. Gross, José Antonio Marcondes, José Augusto Sgarbi, José A. Sisson de Castro, José Gilberto H. Vieira, Josivan Gomes de Lima, Julio Abucham, Jussara V. Toniolo, Karla F.S. Mello, Laércio J. Franco, Laura S. Ward, Léa Z. Maciel, Leão Zagury, Leila B. Araújo, Luciane M. Ribeiro-Neto, Lucila K. Elias, Lucio Vilar, Luis Eduardo Calliari, Luiz Antonio S. Câmara, Luiz Armando de Marco, Luiz Roberto Ramos, Luiza K. Matsumura, Manuel H. AguiarOliveira, Marcello D. Bronstein, Márcio Abrahão, Marcio Mancini, Marco Aurélio Reis, Marcos A. Tambascia, Marcus Aurelho de Lima, Margaret Boguszewski, Margaret de Castro, Maria Adelaide A. Pereira, Maria Alice Bordallo, Maria Candida V. Fragoso, Maria de Fátima Borges, Maria Elisabeth Rossi, Maria Helena Senger, Maria Honorina C. Lopes, Maria Lúcia F. Farias, Maria Lucia Giannella, Maria Teresa Zanella, Maria Tereza Baptista, Maria Tereza Nunes, Marilia de Brito Gomes, Marilia M. Guimarães, Mário A. Saad, Mario Vaisman, Marise Lazaretti-Castro, Martha K. Huayllas, Mauro A. Czepielewski, Mauro Semer, Meyer Knobel, Miguel N. Hissa, Milena C. Fernandes-Caldato, Milton Cesar Foss, Moacir C. de Andrade Jr., Mônica R. Gadelha, Nelson Carvalhaes, Nelson Rassi, Nicolau Lima Neto, Nina R. Musolino, Nuvarte Setian, Omar M. Hauache, Orsine Valente, Osmar Monte, Paulo Tannus Jorge, Pedro Henrique S. Correa, Poli Mara Spritzer, Regina Célia Moisés, Regina do Carmo Silva, Reinaldo P. Furlanetto, Renata Azevedo, Ricardo Meirelles, Roberto B. Bazotte, Rogéria Serakides, Rosa F. dos Santos, Rosalinda Y. Camargo, Rosana Radominski, Rosângela R. Rea, Rosely Sichieri, Rui Curi, Rui Maciel, Ruth Clapauch, Ruy Lyra, Sami Liberman, Sandra $R$. Ferreira, Sandra Villares, Saulo Cavalcanti, Sebastião de Oliveira Jr., Sérgio A. Dib, Sérgio P. Toledo, Silmara O. Leite, Simão A. Lottenberg, Sofia Lemos-Marini, Sonir R.
Antonini, Tânia A.S. Bachega, Thomas Cruz, Turibio L. de Barros Neto, Ubiratan Machado, Vaê Dichchekenian, Valéria Guimarães, Vanda Jorgetti, Vitor Pardini, Walmir Coutinho e Walter Bloise.

Pessoas da maior confiança e "amigos prá toda obra", sou muitíssimo grato aos editorialistas de 2003 2004, que solicitados sempre em cima da hora não deixam jamais de colaborar e entregar seu material dentro do prazo. São eles, em ordem cronológica da publicação: Pedro Henrique S. Corrêa, Poli Mara Spritzer, Cecilia Lacroix de Oliveira, Mauro Fisberg, Geraldo Medeiros-Neto, João Roberto de Sá, César L. Boguszewski, Sabrina M. Coelho, Mário Vaisman, Lucila L.K. Elias, Margaret de Castro, Giuseppe Repetto, Jacqueline Rizzolli, Cassiane Bonatto, Carlos A. Longui, Ana Luiza Maia, Antonio C. Bianco, Valéria Guimarães, João Lindolfo C. Borges, Luiz Cesar Póvoa, Antonio S. Tebexreni, Paola E. Smanio, Eder C. Quintão, Rui Maciel, Léa M. Maciel, Berenice B. Mendonça, Claudio E. Kater, Luis Eduardo Calliari e Rossana Corbo.

As Edições Especiais dos Arquivos, iniciadas em 1998, têm se tornado um atrativo à parte. Tanto a quantidade de material submetido como sua qualidade científica têm sido motivo de muita satisfação para o Conselho Editorial dos ABE\&M. Gostaria, portanto, de agradecer a todos os colaboradores que participaram das Edições Especiais de 2003-2004, na pessoa dos respectivos Editores-Convidados. São eles (por ordem cronológica): César L. Boguszewski ("Neuroendocrinologia”, Agosto de 2003), Ana Luiza Maia e Antonio C. Bianco ("A Glândula Tireóide", Fevereiro de 2004) e Berenice B. de Mendonça e Claudio E. Kater ("Uma Visão Atual das Doenças da Adrenal", Outubro de 2004). Não pude deixar de espreitar a programação de 2005 e divulgar a vocês as duas próximas novidades: "Determinação e Diferenciação Sexual", a ser publicada em Fevereiro de 2005, sob coordenação de Andréa T. Maciel-Guerra e Gil Guerra-Junior e "Oncologia Endócrina”, a sair em Outubro de 2005, sob coordenação de Mauro A. Czepielewski e Jorge L. Gross.

Os anos de 2003 e 2004 foram também repletos de suplementos dos ABE\&M dedicados a Congressos e eventos da especialidade. Assim, gostaria de cumprimentar e agradecer àqueles que de alguma maneira contribuíram para produzir os suplementos dedicados aos seguintes eventos: V COBRAPEM Congresso Brasileiro Pediátrico de Endocrinologia e Metabologia (Abril 2003), V COPEM - Congresso Paulista de Endocrinologia e Metabologia (Abril 2003), ENDO-Recife 2003 e lst Joint Meeting da 
SBEM/AACE (Junho 2003), X CBO - Congresso Brasileiro de Obesidade (Agosto 2003), $14^{\circ}$. Congresso Brasileiro de Diabetes (Novembro 2003), XII SINE Simpósio Internacional de Neuroendocrinologia (Março 2004), VI Congresso Paulista de Diabetes e Metabolismo (Abril 2004), XI EBT - Encontro Brasileiro de Tireóide (Junho 2004), ENDO-Recife 2004 (Julbo 2004) e $26^{\circ}$. Congresso Brasileiro de Endocrinologia e Metabologia (Novembro 2004). Publicamos, também, em Setembro de 2004 (ABE\&M 48/4, suplemento 1) uma edição dedicada aos novos Estatutos e Regimentos da SBEM e seu Cadastro de Sócios.

O patrocínio continuado fornecido por empresas privadas, quer da indústria farmacêutica quer de laboratórios de análises clínicas, através da publicação de anúncios e outros materiais promocionais, tem sido o esteio para a viabilização financeira da revista. Assim, nossos justos agradecimentos, na expectativa da manutenção desse apoio, às seguintes empresas (em ordem alfabética de seus nomes populares, com desculpas pela eventual falha no uso indevido de seus novos nomes, decorrentes de fusões e/ou incorporações recentes: Abbott, Asta Medica, Aventis, Bayer HealthCare, Biosintética, CRIESP, Genzyme, GlaxoSmithKline, Johnsone Johnson, Lilly, Medley, Merck, Novo Nordisk, Pelenova, Pfizer, Pharmacia/ Pfizer, Roche Diagnóstica, Sanofi-Synthelabo, Schering-Plough e Torrent.

Para finalizar, quero fazer alguns outros agradecimentos, dois deles especiais, que vão para Ana Claudia Latronico e Omar M. Hauache, co-editores recentemente escolhidos dos ABE\&M, que têm prestado enorme auxílio "in loco" na divisão de trabalhos e na partilha de decisões da política editorial. O terceiro coeditor convidado, Mário Saad, envolvido que esteve com inúmeras outras funções e encargos junto a órgãos governamentais, não pôde ainda oferecer sua parcela de colaboração que, de comum acordo, deverá ocorrer à partir de 2005. Como as anteriores, a atual Diretoria Nacional da SBEM, sediada em Brasília, merece, na pessoa de Valéria Guimarães, nosso reconhecimento pelo muito que tem feito pela Sociedade e pelos ABE\&M.

As pessoas que trabalham nos bastidores são sempre absolutamente necessárias, se não essenciais para o andamento dos trabalhos, incluindo $o$ cadastramento, organização e processamento dos manuscritos, as correções e revisões, a diagramação e editoração, os filmes, fotolitos e impressão, o levantamento de patrocínio e contratos publicitários, a contabilidade financeira, e a manutenção da ordem e da paz: assim, meu reconhecimento ao Henrique e à Suely (TecArt), à Estela (BlueBox), à Marina (Grámmata), à Roberta e às "meninas" de Brasília, Luciana e Zenaide (secretarias), à Betty (coordenação), à Dorcas e Edeno (SOMED). Também agradeço aos contatos e aos responsáveis pelos interesses dos ABE\&M na BIREME, no LILACS e no SciELO.

Aos nossos prezados colegas, leitores e estimuladores, nosso desejo de um Feliz Natal e um final de ano rico em celebrações e com muita paz. A todos um excelente e promissor 2005. 